\title{
The Impact of Obesity on COVID-19 Disease Severity
}

\author{
Joshua D. Long, BA | Caitlin A. Ward, MPH | Arshia Khorasani-Zadeh, MD
}

PRIMER. 2020;4:15.

Published: 8/14/2020 | DOI: 10.22454/PRiMER.2020.104798

\section{Abstract}

Introduction: Obesity has been declared a major risk factor for morbidity and mortality in COVID-19 patients. In this rapid review, we provide an overview of recently-published papers with clinical and epidemiological relevance on this topic.

Methods: As part of a weekly COVID-19 data mining meeting, we conducted a literature review regarding the role of obesity in COVID-19 outcomes, particularly in young patients with COVID-19. We utilized the PubMed, Upstate Medical University Health Sciences Library, Google Scholar, and LitCovid databases to identify the articles.

Results: Our group identified seven relevant publications (four retrospective case series and three reviews).

Conclusion: Our group's review of this topic illustrates that obesity is a common comorbidity in hospitalized COVID-19 patients. Obesity is associated with an increased likelihood of intermittent mandatory ventilation within the first 10 days of hospitalization and a higher risk of admission to acute or critical hospital care, including in patients aged less than 60 years, with one study showing it to be a greater risk factor than cardiovascular or pulmonary conditions for critical COVID-19 illness. There are some indications that moderate-intensity exercise may be beneficial for promoting a healthy immune system in patients with and without obesity. Given these findings, hospitals should ensure their staff are prepared and their facilities are adequately equipped to provide high-quality care to patients with obesity (PWO) hospitalized with COVID-19. Family medicine and primary care physicians are encouraged to counsel their PWO about their increased risk for morbidity and mortality during this pandemic.

\section{Introduction}

This literature review was conducted as part of a weekly COVID-19 data synthesis at SUNY Upstate Medical University (UMU) that began on March 24, 2020. Our group addressed the topic of this manuscript, along with other COVID-19-related questions during the week of April 27,2020. We aimed to investigate whether obesity is an independent risk factor for morbidity and mortality in patients with COVID-19. In the United States, the prevalence of obesity (BMI $\geq 30)$ and severe obesity (BMI $\geq 40)$ in adults is $42.4 \%$ and $9.2 \%$, respectively. ${ }^{1}$ The Centers for Disease Control and Prevention (CDC) has designated patients with a $\mathrm{BMl} \geq 40$ as high risk for severe illness due to COVID-19. ${ }^{2}$ As social distancing measures are reduced, public health experts anticipate that COVID-19 illness and death will increase. ${ }^{3}$ By understanding the increased risks of COVID-19 for people with obesity (PWO), health care providers can prepare to deliver the care that PWO will require if they contract the illness and require hospitalization.

Previous investigations into this topic include retrospective case series in areas with high rates of COVID-19 cases. In this review, we aimed to compile relevant original research and reviews that would benefit policy makers and health care providers. 


\section{Methods}

During a meeting of our COVID-19 investigation group, we compiled information on whether younger adults with obesity are at higher risk of developing severe symptoms or dying from COVID-19 versus their nonobese peers. Papers were obtained by searching PubMed, the Upstate Medical University Health Sciences Library database, Google Scholar, and the LitCovid database-a database developed by the National Institutes of Health that identifies $35 \%$ more relevant articles than conventional keyword searches for entries on COVID-19 topics. ${ }^{4}$

\section{Results}

We identified seven publications in response to this question. At the time of our literature review on April 27, 2020, most of the available literature on COVID-19 did not stratify outcomes in PWO by age. As a result the manuscripts here also do not. Although there was limited data stratified specifically for young PWO, we were nonetheless able to collect relevant information on PWO infected with SARS-CoV-2 in general, with some insights into how COVID-19 affects younger individuals.

A case series from Northwell Health of 4,170 sequentially hospitalized COVID-19 patients with BMI data found that obesity $(\mathrm{BMl} \geq 30)$ was the second most common comorbidity in the cohort, and $19.0 \%$ of patients were morbidly obese (BMI $\geq 35) .{ }^{5}$ These data provide a glimpse of the prevalence of obesity in hospitalized COVID-19 patients in the United States.

Two retrospective data analyses from the New York University (NYU) health system used data from patients with polymerase chain reaction (PCR)-confirmed COVID-19. The first study included 3,615 sequential COVID-19-positive patients who presented to the emergency department from March 4, 2020 to April 4, 2020. ${ }^{6}$ PWO aged less than 60 years had significantly higher odds of being admitted to acute and critical care compared to individuals under 60 with a BMl<30. For PWO aged less than 60 years with a BMI between $30-34$, these odds were $2.0(95 \% \mathrm{Cl} 1.6-2.6)$ for those admitted to acute care, and $1.8(95 \% \mathrm{Cl} 1.2-2.7)$ for those admitted to critical care. For PWO with BMl $\geq 35$ admitted to acute and critical care, these odds were $2.2(95 \% \mathrm{Cl} 1.7-2.9)$ and $3.6(95 \% \mathrm{Cl} 2.5-5.3)$, respectively. The authors highlighted that obesity may be a risk factor in hospital admission and the need for critical care in patients aged $<60$ years, especially in the United States.

The second study included all COVID-19 patients in the NYU system between March 1, 2020 and April 2, 2020, with follow up through April 7, 2020. ${ }^{7}$ Of 1,999 hospitalized patients, $39.8 \%$ were obese, compared to $14.5 \%$ of nonhospitalized patients. Having a $\mathrm{BMI}>40$ was one of the strongest predictors of hospitalization (OR $6.2,95 \% \mathrm{Cl}$, 4.2-9.3). Obesity was a significant risk factor for critical illness, surpassing any cardiovascular or pulmonary disease (BMI>30 adusted odds ration [AOR] 1.38, 95\% $\mathrm{Cl} 1.03-185$, and BMI>40 AOR 1.73, 95\% $\mathrm{Cl} 1.03-2.9$ ).

A retrospective study from Rhode Island included 103 consecutive adult patients hospitalized with COVID-19. ${ }^{8} \mathrm{~A}$ multivariate regression, which adjusted for age, gender, race, diabetes, heart disease, obesity, severe obesity, and lung disease, found that severe obesity was the only risk factor significantly associated with intensive care unit admission (AOR 5.39, 95\% Cl 1.13, 25.64). Obese (BMI 30-34.9) and severely obese (BMI $\geq 35$ ) individuals were significantly more likely to undergo intermittent mandatory ventilation (IMV) within 10 days of hospitalization (AOR $6.85,95 \% \mathrm{Cl} 1.05,44.82$ and AOR 9.99, 95\% Cl 1.39, 71.69, respectively).

We included two reviews that looked at the possible benefits of aerobic exercise in preventing or mitigating the effects of COVID-19.9,10 Both reviews suggest that moderate exercise should be recommended to boost immune system function during the COVID-19 pandemic. However, high intensity exercise may induce an inflammatory response and suppress the immune system if the individual participates in prolonged bouts of intensive exercise. This inflammatory response is thought to be caused by an increase in certain proteins, including serum amyloid A-4, myeloperoxidase, and plasma protease $\mathrm{C} 1$ inhibitor that increase during the recovery phase after intense exercise. ${ }^{11}$

Another review included in the literature search assessed mechanistic problems involved with PWO and COVID-19, including the limited number of bariatric beds in hospitals; difficulty with adjusting and transporting patients in these 
beds; possibly compromised imaging due to machine weight limits; and difficulty with intubation in PWO. ${ }^{12}$ The authors also suggest that the chronic inflammation caused by obesity is similar to the increased inflammatory response seen in COVID-19.

The results of this review, while brief, indicate that PWO who became infected with SARS-CoV-2 during the initial wave of the pandemic in the Northeastern United States experienced worse outcomes compared to nonobese individuals. Additionally, our review highlights the unique and significant logistical and procedural barriers that PWO and their physicians may confront when PWO with COVID-19 are admitted to the hospital. Our review of the literature also suggests that moderate exercise may support immune system health, which in turn may decrease the risk of patients developing severe symptoms if they become infected with COVID-19.

\section{Conclusions}

Our review illustrates that in addition to being a common comorbidity in hospitalized COVID-19 patients, obesity has been associated with a higher risk of admission to acute or critical hospital care, including in patients younger than 60 years of age; higher rates of IMV within the first 10 days of hospitalization; and is a risk factor for critical illness due to COVID-19.

Based on the findings of this review and CDC guidance that $B M I \geq 40$ is a risk factor for severe COVID-19 illness, hospitals should strategize how to best utilize their expertise and supply chain to serve this patient population. Clinicians should consider encouraging exercise to all patients interested in taking an active role in potentially boosting their immune system. Policy makers should consider these findings when developing policies to reopen their communities.

Patients in medically high-risk groups are currently being counseled to modify their daily routines to stay safe during this pandemic. These data demonstrate that family medicine and primary care providers can play a key preventative role in the response to this pandemic by counseling PWO in routine visits (including through telemedicine) about their increased risk of COVID-19-related morbidity and mortality. Informing these patients that they are included in a high-risk group may further encourage them to avoid situations and environments with high infection risk.

This review has a number of limitations. This was not a systematic review. It should be viewed as a brief overview of relevant publications for clinicians and policy makers. Future research should include meta-analyses and retrospective data analyses from more geographically diverse patient populations. Additionally, stratifying outcomes by age among PWO would provide more insight into whether young PWO are an at-risk group in the COVID-19 pandemic.

\section{Acknowledgments}

The authors thank Kate Bunch and Nick Bender, third-year medical students from SUNY Upstate Medical University College of Medicine, for their literature review contributions to this manuscript.

\section{Corresponding Author}

Joshua D. Long, BA

SUNY Upstate Medical University, College of Medicine, 750 East Adams Street, Syracuse, NY 13210-2375. longjo@upstate.edu

\section{Author Affiliations}

Joshua D. Long, BA - SUNY Upstate Medical University, College of Medicine, Syracuse, NY

Caitlin A. Ward, MPH - SUNY Upstate Medical University, College of Medicine, Syracuse, NY

Arshia Khorasani-Zadeh, MD - Department of Medicine, SUNY Upstate Medical University 


\section{References}

1. Hales CM, Carroll MD, Fryar CD, Ogden CL. Prevalence of Obesity and Severe Obesity Among Adults: United States, 2017-2018 Key Findings Data from the National Health and Nutrition Examination Survey.; 2017. https://www.cdc.gov/nchs/products/index.htm. Accessed May 11, 2020.

2. Centers for Disease Control and Prevention. People Who Are at Higher Risk for Severe Illness https://www.cdc.gov/coronavirus/2019-ncov/need-extra-precautions/people-at-higher-risk.html. Published 2020. Accessed May 12, 2020.

3. Matrajt L, Leung T. Evaluating the Effectiveness of Social Distancing Interventions to Delay or Flatten the Epidemic Curve of Coronavirus Disease. Emerg Infect Dis. 2020;26(8):1740-1748. doi:10.3201/eid2608.201093

4. Chen Q, Allot A, Lu Z. Keep up with the latest coronavirus research. Nature. 2020;579(7798):193. doi:10.1038/d41586-020-00694-1

5. Richardson S, Hirsch JS, Narasimhan M, et al; Northwell COVID-19 Research Consortium. Presenting characteristics, comorbidities, and outcomes among 5700 patients hospitalized with COVID-19 in the New York City area. JAMA. 2020;323(April):2052. doi:10.1001/jama.2020.6775

6. Lighter J, Phillips M, Hochman S, et al. Obesity in patients younger than 60 years is a risk factor for COVID-19 hospital admission. Clin Infect Dis. 2020;(ciaa415). doi:10.1093/cid/ciaa415

7. Petrilli Christopher M, Jones Simon A, Yang Jie, Rajagopalan Harish, O'Donnell Luke, Chernyak Yelena et al. Factors associated with hospital admission and critical illness among 5279 people with coronavirus disease 2019 in New York City: prospective cohort study BMJ. 2020; 369 :m1966

8. Kalligeros M, Shehadeh F, Mylona EK, Benitez G, Beckwith CG, Chan PA, Mylonakis E. Association of obesity with disease severity among patients with coronavirus disease 2019. Obesity. 2019. 28:1200-1204. doi:10.1002/oby.22859

9. Rahmati-Ahmadabad S, Hosseini F. Exercise against SARS-CoV-2 (COVID-19): does workout intensity matter? (A mini review of some indirect evidence related to obesity). Obes Med. 2020;100245(April):100245. doi:10.1016/j.obmed.2020.100245

10. Mohamed AA, Alawna M. Role of increasing the aerobic capacity on improving the function of immune and respiratory systems in patients with coronavirus (COVID-19): A review. Diabetes Metab Syndr. 2020;14(4):489-496. doi:10.1016/j.dsx.2020.04.038

11. Nieman DC, Wentz LM. The compelling link between physical activity and the body's defense system. J Sport Health Sci. 2019;8(3):201-217. doi:10.1016/j.jshs.2018.09.009

12. Michalakis $\mathrm{K}$, llias I. SARS-CoV-2 infection and obesity: common inflammatory and metabolic aspects. Diabetes Metab Syndr. 2020;14(4):469-471. doi:10.1016/j.dsx.2020.04.033 